\title{
Funcionamento patológico da personalidade para predição da depressão, qualidade de vida e esperança
}

\author{
André Pereira Gonçalves \\ Ruam Pedro Francisco de Assis Pimentel \\ Lucas de Francisco Carvalho
}

\section{RESUMO}

Estudos prévios apontam que níveis de funcionamentos patológico da personalidade (FPP) se associam com maior presença de sintomas depressivos, baixa qualidade de vida e esperança. Entretanto, não foram observados estudos utilizando conjuntamente essas três variáveis em um modelo focado no FPP. 0 objetivo deste trabalho foi verificar a capacidade preditiva dos níveis de FPP sobre qualidade de vida (QV), esperança e depressão. Participaram 475 sujeitos com idade entre 18 a 70 anos e em sua maioria mulheres $(81,1 \%)$, que responderam aos seguintes instrumentos: Inventário Dimensional Clínico versão triagem (IDCP-triagem), Escala Baptista de depressão versão triagem (EBADEP-A-triagem), WHOQOL-Bref e Escala de Esperança de Herth (EEH). Por meio da modelagem de equações estruturais, indicadores de depressão foram positivamente preditos pelo FPP, enquanto os de QV e esperança foram negativamente preditos, tal qual as hipóteses do estudo. Estes achados demonstram a alta capacidade preditiva do FPP sobre depressão, QV e esperança. Na clínica, ao observar perfil que combine estas características pode ser indicado a necessidade da avaliação dos aspectos patológicos da personalidade.

Palavras-chave: avaliação da personalidade; transtornos da personalidade; avaliação psicológica.

\section{ABSTRACT}

\section{Pathological functioning of personality for prediction of depression, quality of life and hope}

Previous studies suggest that more severe levels of personality (i.e., personality pathological functioning - PPF) are associated to the presence of depressive symptoms, low quality of life, and hope. However, we could not find publications using at the same time these three variables in a model focused on the FPP. The aim of this study was to verify the predictive capacity of the levels of pathological personality functioning (PPF) on quality of life (QL), hope and depression. Participants were 475 subjects, aged between 18 and 70 years, mostly women (81.1\%), who responded to the Dimensional Clinical Personality Inventory screening version (IDCP-screening), Baptista Depression Rating Scale (EBADEP-A-screening), WHOQOL-Bref, and Herth Hope Scale (EEH). Through the structural equation modeling, depression indicators were positively predicted by PPF, while those of QL and hope were negatively predicted, according to the study's hypothesis. These findings demonstrate the high predictive capacity of the PPF on depression, QL, and hope. We conclude that clinicians should consider to assess pathological aspects of the personality when observing a profile that combines high levels of depression and low levels of QL and hope.

Keywords: personality assessment; personality disorders; psychological assessment.

Partindo da visão de uma abordagem dimensional o nível de funcionamento da personalidade é entendido enquanto um continuum entre o saudável e o patológico, ou seja, o mesmo conjunto de traços estão presentes em todos sujeitos, e o que varia é a intensidade da manifestação destes traços (American Psychiatric Association, 2014). Na direção dos aspectos

\section{Sobre os Autores}

A. P. G.

orcid.org/000-0002-2470-4040 Universidade São Francisco

(USF) - Campinas, SP

andregoncalvespsi@gmail.com

R. P. F. A. P.

orcid.org/0000-0001-6106-2342 Universidade São Francisco (USF) - Campinas, SP ruampimentel@gmail.com

L. F. C.

orcid.org/000-0002-3274-9724

Universidade São Francisco

(USF) - Campinas, SP

lucas@labape.com.br

\section{Direitos Autorais}

Este é um artigo de acesso aberto e pode ser reproduzido livremente, distribuído,

transmitido ou modificado, por qualquer pessoa desde que usado sem fins comerciais. 0 trabalho é disponibilizado sob a licença Creative Commons CCBY-NC.

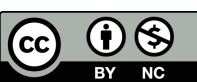


saudáveis, nota-se características e comportamentos presentes em grande parte da população, enquanto na direção do funcionamento patológico da personalidade (FPP), observase padrões persistentes e mal adaptativos que desviam das expectativas socioculturais do grupo de pertencimento de um sujeito (Millon, 2011; Widiger \& Costa, 2013).

Três características globais configuram o FPP (Millon, 2011): baixa resiliência, baixa flexibilidade adaptativa e círculo vicioso. A baixa resiliência refere-se à capacidade diminuída em lidar com situações estressoras do cotidiano; a baixa flexibilidade adaptativa diz respeito à incapacidade de criar novos mecanismos que auxiliem em determinadas situações; e o círculo vicioso é caracterizado pela dificuldade do indivíduo para mudar os padrões de funcionamento, tornando-os persistentes.

O FPP se refere à manifestação patológica de diversos traços da personalidade, caracterizando um padrão patológico. O FPP deve ser compreendido em um continuum dimensional, isto é, ele pode ser apresentado em diferentes níveis de severidade, o que pode implicar prejuízos nas relações interpessoais (i.e., relacionamento com outro) e relações com o self (i.e., relação da pessoa com ela mesma; Skodol et al., 2011; Widiger \& Costa, 2013). Embora as causas específicas que levam a diferentes pessoas apresentar níveis diferentes de FPP sejam desconhecidas, sabe-se que fatores biológicos/ genéticos, sociais/aprendizagem e psicológicos impactam na manifestação dos traços de personalidade (Millon, 2011). Indivíduos com níveis mais severos do FPP são usualmente classificados e categorizados em diferentes quadros psicopatológicos (Millon, 2011), especificamente, transtornos da personalidade (TP; American Psychiatric Association, 2014).

Evidências prévias indicam que níveis mais altos de FPP estão relacionados com diversos fatores de risco, como sintomas depressivos (Fava et al., 1996; Sevilla-Llewellyn-Jones et al., 2018; Tsanas et al., 2016), e fatores protetivos, como a qualidade de vida (Boye et al., 2008; Cramer et al., 2006; Crempien et al., 2017; Filipović et al., 2013), e a esperança (Bayrami et al., 2012; Chioqueta \& Stiles, 2005; Göktan \& Akbağ, 2010; Jonason et al., 2018; Mutlu et al., 2010). Com base nos achados prévios, nosso foco no presente estudo é testar um modelo empírico, investigando a capacidade preditiva dos níveis de FPP sobre sintomas depressivos, qualidade de vida e esperança.

A depressão é compreendida enquanto sintomatologia frequentemente presente em transtornos de humor, e ainda enquanto um conjunto de sintomas que recebe seu próprio status de transtorno depressivo em que anedonia e alterações de humor são principais sintomas (American Psychiatric Association, 2014). Estudos vêm demonstrando relações positivas entre sintomas de depressão e FPP (Fava et al., 1996;
Sevilla-Llewellyn-Jones et al., 2018; Tsanas et al., 2016). Van der Wal et al., (2018) encontraram que pessoas diagnosticadas com TPs apresentaram maior risco de desenvolver transtorno depressivo maior quando comparadas com grupo de não pacientes. Além disso, evidências prévias indicaram correlações positivas entre a severidade dos sintomas depressivos e os níveis de FPP (Crempien et al., 2017).

A qualidade de vida, definida como a percepção dos indivíduos de suas posições de vida no contexto da cultura e sistema de valores nos quais vivem e na relação com seus objetivos, expectativas, padrões e preocupações (WHOQOL GROUP, 1998), parece também ser impactada pelo FPP. Por exemplo, elevados níveis dos traços de neuroticismo podem levar o sujeito à percepção dos aspectos mais negativos da sua vida e consequentemente um sentimento empobrecido quanto a sua QV (Boye el tal., 2008; Crempien et al., 2017; Filipović et al., 2013). Cramer et al.(2006) encontraram que a o FPP têm maior poder preditivo para empobrecimento da QV do que variáveis sociodemográficas, e transtornos psiquiátricos. Esses achados sugerem que níveis mais altos de FPP se relaciona com baixa QV.

A esperança pode ser definida como a crença em um futuro próspero e favorável, e está relacionada com preocupações quanto às ações iniciadas por si que possam criar um futuro com mais possibilidade de sucesso (Alarcon et al., 2013). Foram encontrados poucos estudos que relacionam esperança e níveis de FPP utilizando como descritores os termos personalidade e esperança em português e inglês no Google Acadêmico. Os estudos encontrados focam na relação com a baixa esperança (i.e., desesperança), indicando relações positivas entre FPP e baixos níveis de esperança/desesperança (Bayrami et al., 2012; Chioqueta \& Stiles, 2005; Göktan \& Akbağ, 2010; Jonason et al., 2018; Mutlu et al., 2010). Considerando os achados prévios, as hipóteses sobre níveis de esperanças foram formuladas a partir de estudos focando na desesperança.

Achados prévios também sugerem que sintomas depressivos, QV e esperança (ou desesperança) se correlacionam entre si: correlações negativas entre QV e depressão (Abrams et al., 2001; Crempien et al., 2017), correlações negativas entre depressão e esperança (Schrank et al., 2014), e correlações positivas entre esperança e QV (Vrbova et al., 2017).

Em síntese, os estudos apresentados indicam que o nível de FPP tende a se relacionar com sintomas depressivos, QV e esperança. $O$ objetivo deste trabalho é verificar a capacidade preditiva dos níveis de FPP sobre QV, esperança e depressão por meio de modelagem de equações estruturais. Para prosseguir com as análises para verificação da capacidade preditiva do nível de FPP sobre as variáveis dependentes, verificar-se-á as estruturas fatoriais das medidas utilizadas 
no estudo. Com base nos estudos que foram apresentados até o momento, este trabalho tem as seguintes hipóteses: (H1) a estrutura fatorial dos instrumentos de medidas deve apresentar bons índices de ajustes e carga fatorial $\geq 0,30$ (Baptista \& Carvalho, 2018; Carvalho et al., 2017; Sartore \& Grossi, 2008; WHOQOL-Group, 1998); (H2) o nível de FPP terá capacidade preditiva significativa e positiva sobre depressão (Fava et al., 1996; Sevilla-Llewellyn-Jones et al., 2018; Tsanas et al., 2016); (H3) o nível de FPP terá capacidade preditiva significativa e negativa sobre os níveis de QV (Boye et al., 2008; Cramer et al., 2006; Crempien et al., 2017; Filipović et al., 2013); (H4) nível de FPP terá capacidade preditiva significativa e negativa sobre os níveis de esperança (Bayrami et al., 2012; Chioqueta \& Stiles, 2005; Göktan \& Akbağ, 2010; Jonason et al., 2018; Mutlu et al., 2010).

\section{MÉTODO}

\section{PARTICIPANTES}

Um total de 475 participantes foram incluídos neste estudo, com idade entre 18 e 70 anos ( $M=34,2 ; S D=11,88)$ em sua maioria mulheres $(81,1 \%)$ e brancos $(72,4 \%)$. A respeito do nível escolar, $26,9 \%$ possuem nível superior completo, $23,6 \%$ finalizaram o ensino médio e $21,3 \%$ possuem curso superior incompleto. Dentre os participantes, $8,2 \%$ reportaram já ter feito tratamento psiquiátrico, $13,1 \%$ tratamento psicológico e $31,6 \%$ relataram em algum momento ter apresentado ideação suicida.

\section{INSTRUMENTOS}

\section{Inventário Dimensional Clínico da Personalidade - triagem (IDCP-triagem; Carvalho et al., 2017)}

O IDCP-triagem foi desenvolvido com base na versão completa do IDCP (Carvalho \& Primi, 2015), um teste com intuito de mensurar traços patológicos de personalidade. 0 instrumento consiste em 15 itens em uma escala tipo Likert de 4 pontos, em que 1 corresponde a "nada a ver comigo" e 4 "tudo a ver comigo". Para este estudo não foi utilizado a variável dicotômica do instrumento, e sim a variável escalar como um indicador de personalidade patológica, em que os mais altos escores indicam um funcionamento mais patológico. A escala foi capaz de discriminar grupos com transtorno de personalidade e grupo sem transtornos de personalidade com sensibilidade 89,5 e especificidade de 67,2. Estudos demostram adequação das propriedades psicométricas do IDCP-triagem (Carvalho et al., 2017; Carvalho, 2018). A análise de consistência interna demonstrou boa adequação do instrumento $(a=0,85)$ com a amostra do presente estudo.

\section{Escala Baptista de Depressão - Versão Triagem [EBADEP- triagem; Baptista \& Carvalho, 2018]}

A EBADEP-triagem foi desenvolvida com base na versão adulta do EBADEP (EBADEP-A; Baptista, 2012) e tem como objetivo avaliar os sintomas da depressão. Na EBADEP-triagem, foram selecionados 15 itens relacionados ao humor triste, anedonia, culpa, fadiga, concentração, ideação suicida e sono. No estudo de desenvolvimento, a EBADEP-triagem foi capaz de discriminar 40 pacientes diagnosticados com depressão por SCID-I de 40 pessoas sem depressão, com sensibilidade igual a 95,0 e especificidade de 87,5. Na amostra do presente estudo, a fidedignidade por consistência interna da EBADEP-triagem foi igual a 0,88.

\section{Instrumento de avaliação da qualidade de vida da Organiza- ção Mundial da Saúde (WHOQOL-Bref; WHOQOL GROUP, 1998)}

WHOQOL-bref é a versão reduzida do questionário completo WHOQOL. 0 instrumento tem como objetivo avaliar a qualidade de vida, composto por 26 itens com pontuação em escala Likert de 5 pontos, em que 1 refere-se a "nada satisfeito" e 5 "muito satisfeito". Os itens são subdivididos 4 dimensões, a saber, Físico, Psicológico, Relações Sociais e Meio Ambiente. O domínio físico refere-se a dor e desconforto, energia e fadiga, sono e repouso, atividades da vida cotidiana, dependência de medicação ou de tratamentos, capacidade de trabalho. O Psicológico diz respeito a sentimentos positivos, pensar, aprender, memória de concentração, autoestima, imagem corporal, crenças pessoais. As Relações Sociais referem-se às relações pessoais, atividade sexual, apoio social. E o domínio Meio Ambiente diz respeito à segurança física e proteção, ambiente no lar, recursos financeiros, disponibilidade e qualidade, oportunidades de adquirir novas informações e habilidades, participação em oportunidades de recreação/ lazer; ambiente físico (poluição, ruído, clima), transporte. Neste estudo a fidedignidade por consistência interna variou entre 0,66 (domínio Físico) e 0,84 (Relações Sociais). Uma vez que não foi utilizado o escore total do instrumento, foram considerados somente os 24 itens que compõem as quatro dimensões e não incluindo os dois itens da escala geral. Estudos buscando evidência de validade nas variáveis internas e com bases nas variáveis externas mostram-se adequados (Castro et al., 2013).

\section{Escala de Esperança de Herth [Sartore \& Grossi, 2008)}

Trata-se de uma escala de autorrelato de origem norteamericana (Herth, 1992), com objetivo de quantificar a esperança de vida. 0 instrumento possui 12 afirmativas e a graduação dos itens segue a escala tipo Likert de quatro pontos, 
variando de 1 "concordo completamente" a 4 "discordo completamente". Os itens são distribuídos em três fatores, a saber, Temporalidade e Futuro que se referem à presença de objetivos e perspectiva positiva da vida; Prontidão e Expectativas Positivas diz respeito à percepção de saídas e senso de direção; Relacionamento Consigo e com Outro, refere-se ao sentimento de esperança direcionado a si e aos outros. 0 escore total varia de 12 a 48 e quanto maior o escore, mais alto o nível de esperança de vida. No presente estudo a consistência interna variou entre 0,77 (relacionamento consigo e com outros) e 0,81 (expectativas positivas). Essa escala apresenta validade de construto adequado, a partir de estudos com evidências de validade na estrutura interna e com base em variáveis externas (Sartore \& Grossi, 2008).

\section{PROCEDIMENTOS}

Este estudo foi enviado e posteriormente aprovado por um Comitê de Ética em Pesquisa da Universidade São Francisco (CAAE: 4829618.5.0000.5514). A coleta foi realizada via Google Formulários, de modo que os participantes foram convidados por e-mail, Facebook e pelo aplicativo WhatsApp. No Facebook, o link foi enviado para contatos dos pesquisadores além de postagens em grupos diversificados. No WhatsApp o link foi enviado aos contatos dos pesquisadores e postados em grupos diversificados. Ao acessar o link da pesquisa, era apresentado a versão digital do Termo de Consentimento Livre e Esclarecido e, somente após leitura e anuência, a pesquisa foi liberada ao participante. 0 tempo aproximado para responder foi de 25 minutos.

\section{ANÁLISE DE DADOS}

Para análise dos dados foram utilizados os programas SPSS 21 e MPLus 7. Foi realizada a modelagem de equações estruturais, em que o nível de FPP é a variável independente; enquanto qualidade de vida, esperança e depressão são as variáveis dependentes. Considerando o nível ordinal das variáveis, foi utilizado o estimador ELSMV, que utiliza a matriz de correlação policórica. As variáveis latentes foram estimadas por uma análise fatorial confirmatória com a utilização de todos os itens, considerando critério mínimo cargas fatorais maiores a partir de 0,30 (Hair Jr. et al., 2005). A consistência interna foi obtida por meio do Alfa de Cronbach. 0 nível de FPP foi determinado com uma única variável independente no modelo, enquanto para as variáveis dependentes foram utilizados depressão (indicador geral), os fatores da escala de qualidade de vida (WHOQOL-Bref; Físico, Psicológico, Relações Sociais e Meio Ambiente) e os fatores da Escala de Esperança de Herth (EEH; Temporalidade e Futuro, Prontidão e Expectativas Positivas, Relacionamento Consigo e com Outro). Com intuito de verificar o ajuste do modelo, foram utiliza- dos os seguintes índices: Qui-quadrado (X2/df < 3), Confirmatory Fit Index (CFI; > .90), Tucker-Lewis Index (TLI; > . 90) e Root Mean Square Error of Approximation (RMSEA; < . 06), de acordo com os critérios apontados por Hu e Bentler (1999) e Hair Jr. et al. (2005).

\section{RESULTADOS}

A análise fatorial confirmatória visou investigar se as estruturas fatoriais indicadas na literatura se mantinham nesta amostra. Para o IDCP-triagem verificou-se que os itens se agruparam em um fator com carga fatorial de 0,20 a 0,92, com apenas um item com carga inferior a 0,30 e a variância residual apresentou valores entre 0,14 e 0,96. A EBADEP-triagem esteve dentro do esperado com um fator, com cargas entre 0,43 a 0,86 com variância residual entre 0,26 e 0,81. A escala WHOQOL-bref apresentou adequação aos quatro fatores (Físico, Psicológico, Relações Sociais e Meio Ambiente), com carga variando de .43 a .96 e variância residual entre 0,07 a 0,81 . A EEH adequou-se ao modelo de três fatores, com cargas entre .65 a .89 e variância residual entre 0,19 a 0,49 . Os índices de ajuste do modelo foram todos satisfatórios $(\mathrm{X} 2 / \mathrm{df}=2,15 ; \mathrm{CFI}=0,94 ; \mathrm{TLI}=0,94$; RMSEA = 0,04), demonstrando ajuste aos dados. $\mathrm{Na}$ Figura 1 estão apresentadas as regressões por meio de modelagem de equação estrutural, com intuito de avaliar a capacidade preditiva FPP sobre as variáveis dependentes deste estudo (i.e., qualidade de vida, esperança e depressão).

É possível verificar na Figura 1 que o nível FPP explicou de forma negativa todos os fatores de WHOQOL-bref (Físico, Psicológico, Relacionamentos Sociais e Meio Ambiente), e também com os fatores EEH (Temporalidade e Futuro, Expectativas Positivas, Relacionamento Consigo e com os Outros). Já o escore de depressão foi negativamente predito pelo nível de FPP. O fator psicológico da WHOQOL-bref, o fator relacionamentos consigo e com os outros da EEH e o escore de depressão foram aos mais explicados pela variável independente. Estes dados sugerem que, quanto mais traços patológicos da personalidade, menores os níveis de esperança e qualidade de vida, e maiores níveis de depressão.

\section{DISCUSSÃO}

O objetivo deste estudo foi verificar a capacidade preditiva dos níveis de FPP sobre qualidade de vida, esperança e depressão. Como etapa anterior foi realizada a avaliação da estrutura fatorial como pressuposto para prosseguimento com as análises. Os resultados encontrados corroboram as quatro hipóteses deste estudo. Eram esperados bons índices de ajuste $(\mathrm{H} 1)$, cargas fatoriais $\geq 0,30$ e consistência interna $\geq$ 


\section{W'INTERACÃO EM ET PSICOLOGIA}

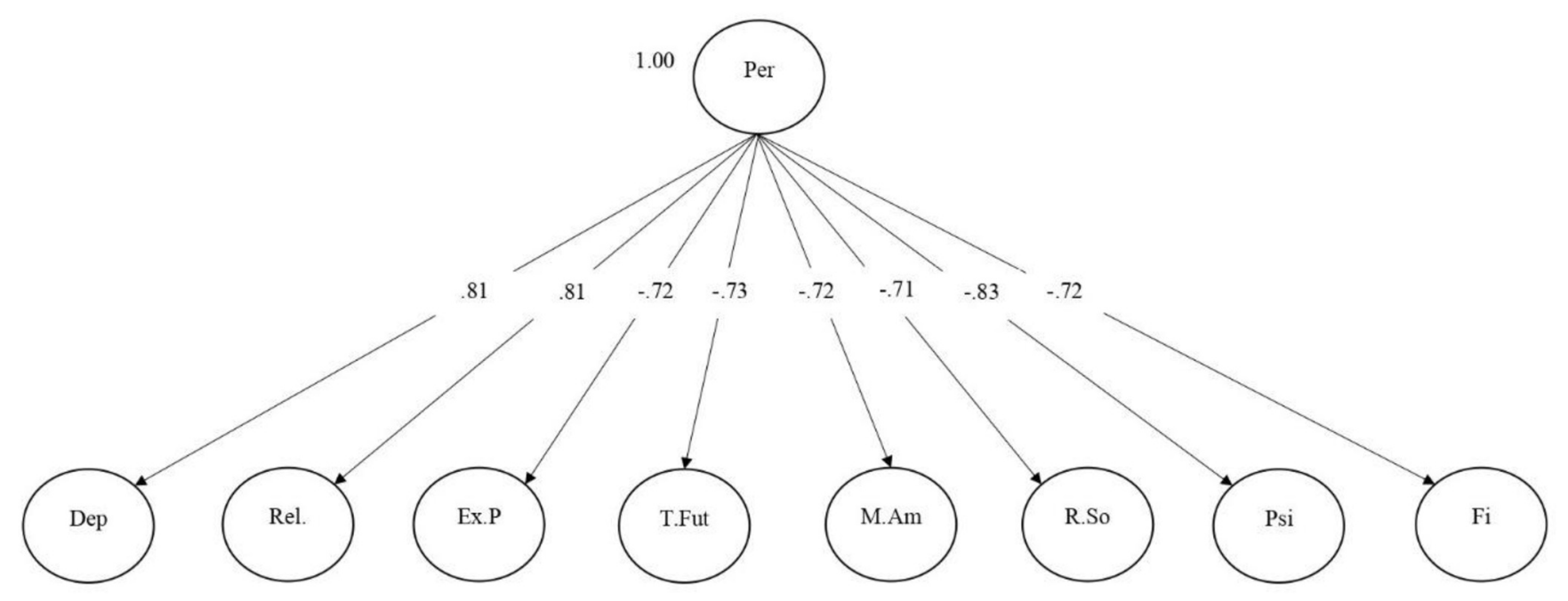

Figura 1. SEM do indicador de personalidade patológica sobre qualidade de vida, esperança e depressão. Nota. Per = Personalidade; Fi = Físicos; Psi = Psicológico; R.So. = Relações Sociais; M. Am = Meio Ambiente; T.Fut = Temporalidade e Futuro; Exp.P = Expectativa Positiva; Rel. = Relacionamento Consigo e com outros; Dep = Depressão

0,70 (Baptista \& Carvalho, 2018; Carvalho et al., 2017; Sartore \& Grossi, 2008; WHOQOL-Group, 1998); além disso, o nível de FPP foi capaz de predizer positivamente a depressão $(\mathrm{H} 2)$ (Fava et al., 1996; Sevilla-Llewellyn-Jones et al., 2018; Tsanas et al., 2016) e negativamente a qualidade de vida (H3) (Boye et al., 2008; Cramer et al., 2006; Crempien et al., 2017; Filipović et al., 2013) e esperança (H4) (Bayrami et al., 2012; Chioqueta \& Stiles, 2005; Göktan \& Akbağ, 2010; Jonason et al., 2018; Mutlu et al., 2010). A seguir são apresentados os resultados de forma detalhada.

A análise fatorial confirmatória foi realizada com intuito de verificar se, com a amostra deste estudo, os instrumentos manteriam os itens fixados carregando nos fatores, conforme a literatura. Todos os instrumentos utilizados confirmaram a literatura apresentando cargas fatoriais satisfatórias e ajuste adequado para o modelo testado (Hair Jr. et al., 2005; Hu \& Bentler, 1999). O IDCP-triagem apresentou apenas um item com carga inferior ao critério utilizado, mas os demais itens se agruparam em um fator como esperado (Carvalho et al., 2017). A escala de qualidade de vida, WHOQOL-Bref, apresentou cargas fatoriais confirmando o modelo com quatro fatores da versão original (Físico, Psicológico, Relações Sociais e Meio Ambiente; WHOQOL Grup, 1998). No que se refere à $\mathrm{EEH}$, foi possível confirmar o modelo com três fatores, com índices satisfatórios (Temporalidade e Futuro, Prontidão e Expectativas Positivas, Relacionamento Consigo e com outro (Sartore \& Grossi, 2008). Por fim, a EBADEP-triagem apresentou cargas satisfatórias para o modelo com apenas um fator (Baptista, 2012).
As análises das regressões obtidas por meio da modelagem de equações estruturais mostraram que a melhor capacidade preditiva dos níveis FPP foi sobre depressão. Estudos prévios demonstram que pessoas com níveis FPP tendem a apresentar maiores índices depressivos (Alizadeh et al., 2017; Crempien et al., 2017; Kotov et al., 2010; Robison et al., 2009; van der Wal et al., 2018). Além disso, dentro do conjunto de traços que caracterizam alguns TPs (Dependente, Evitativo, Esquizoide, Histriônico), a depressão está presente como um fator importante para a caracterização do transtorno (American Psychiatric Association, 2014; Kotov et al., 2017).

Os níveis de FPP apresentaram capacidade preditiva negativa de qualidade de vida, indicando que pessoas com traços mais patológicos têm menor percepção de qualidade de vida do que pessoas com menores indicadores, corroborando os achados na literatura da área (Boye et al., 2008; Crempien et al., 2017; Filipović et al., 2013; Penedo et al., 2003). A percepção de qualidade de vida que mais foi explicada pelos níveis FPP foi o fator psicológico quando comparado ao fator físico, relacionamentos sociais e meio ambiente. Este achado pode estar relacionado com as características avaliadas pelo fator psicológico, que avalia a qualidade de vida frente aos sentimentos positivos e negativos, autoestima, imagem corporal, aparência e crenças pessoais (WHOQOL GROUP, 1998).

No que diz respeito à esperança, níveis de FPP mais altos foram capazes de predizer negativamente esse construto, confirmando achados prévios, que relacionam o nível de FPP com maior desesperança (Bayrami et al., 2012; Chioqueta \& Stiles, 2005; Marco et al., 2017; Mutlu et al., 2010). Além dis- 
so, níveis mais extremos de FPP são representados pelos TPs, que no DSM-5 estão relacionados com a baixa esperança (American Psychiatric Association, 2014).

Os resultados do presente estudo fornecem dados que possibilitam o entendimento de que os níveis de FPP possuem alta capacidade preditiva sobre qualidade de vida, esperança e depressão. No contexto clínico, ao se observar um perfil que combine indicadores de depressão associados a relatos de baixa qualidade de vida e desesperança, pode ser um indicativo para o clínico investigar o funcionamento da personalidade em busca de aspectos patológicos.

Este estudo apresenta algumas limitações que precisam ser consideradas. A amostra contou, em sua grande maioria, com pessoas do sexo feminino (80\%), o que pode enviesar os resultados quanto à generalização, portanto, sugere-se que estudos com pareamento da amostra quanto ao sexo sejam realizados. Outra possível limitação deste estudo foi que o instrumento para avaliação do FPP não abrange todos os aspectos do critério A da seção III do DSM-5. Sugere-se ainda que estudos com objetivo de buscar relações do nível de FPP com outras variáveis dependentes sejam realizados e utilizando um modelo com variáveis moderadoras e/ou mediadoras.

\section{DECLARAÇÃO DA CONTRIBUIÇÃO DOS AUTORES}

A.P.G. realizou a análise formal dos dados e tabulação dos dados; A.P.G. e R.P.F.A.P. foram responsáveis pela redação inicial (rascunho original), conceitualização da temática e redação final (revisão e edição); L.F.C. foi o responsável pela administração do projeto, investigação e supervisão do estudo.

\section{DECLARAÇÃO DE CONFLITOS DE INTERESSE}

Os autores declaram que não há conflitos de interesse no manuscrito submetido.

\section{REFERÊNCIAS}

Abrams, R. C., Alexopoulos, G. S., Spielman, L. A., Klausner, E., \& Kakuma, T. (2001). Personality disorder symptoms predict declines in global functioning and quality of life in elderly depressed patients. The American Journal of Geriatric Psychiatry, 9(1), 67-71. https://doi.org/ 10.1097/00019442-200102000-00010

Alarcon, G. M., Bowling, N. A., \& Khazon, S. (2013). Great expectations: A meta-analytic examination of optimism and hope. Personality and Individual Differences, 54(7), 821827. https://doi.org/10.1016/j.paid.2012.12.004
Alizadeh, Z., Feizi, A., Rejali, M., Afshar, H., Keshteli, A. H., \& Adibi, P. (2017). The predictive value of personality traits for psychological problems (stress, anxiety and depression): Results from a large population based study. Journal of Epidemiology and Global Health, 8(3-4), 124-133. https:// doi.org/10.1016/j.jegh.2017.11.003

American Psychiatric Association APA. (2014). Manual dianóstico e estatístico de trantornos mentais (5o ed). Artmed.

Baptista, M. N. (2012). Manual técnico da Escala Baptista de Depressão em Adultos (EBADEP-A)[Baptist Adult Depression Scale Technical Manual]. Vetor.

Baptista, M. N., \& Carvalho, L. F. (2018). Diagnostic accuracy of a Brazilian depression self-report measure (EBADEP): Original and short versions. Avaliação Psicológica, 17(4), 484-492. http://dx.doi.org/10.15689/ap.2018.1704.8.08

Bayrami, M., Heshmati, R., Ghotbi, M., Ghoradel, J. A., Hojatipor, H., \& Moslemifar, M. (2012). Relationship between personality dimensions and hopelessness: A study on college students. Procedia - Social and Behavioral Sciences, 46, 848-852. https://doi.org/10.1016/j.sbspro. 2012.05.211

Boye, B., Lundin, K. E. A., Leganger, S., Mokleby, K., Jantschek, G., Jantschek, I., Kunzendorf, S., Benninghoven, D., Sharpe, M., Wilhelmsen, I., Blomhoff, S., Malt, U. F., \& Jahnsen, J. (2008). The INSPIRE study: Do personality traits predict general quality of life (Short form-36) in distressed patients with ulcerative colitis and Crohn's disease? Scandinavian Journal of Gastroenterology, 43(12), 1505-1513. https://doi.org/10.1080/00365520802321196

Carvalho, L. F. (2018). Review study of the impulsiveness dimension of the Dimensional Clinical Personality Inventory. Universitas Psychologica, 17(1), 58-68. https://doi.org/ 10.11144/javeriana.upsy17-1.rsid

Carvalho, L. F., Pianowski, G., \& Reis, A. M. (2017). Development and diagnostic accuracy of the screening of the Dimensional Clinical Personality Inventory. Psicologia: Ciência e Profissão, 37(4), 1011-1024. https://doi.org/ 10.1590/1982-3703003082016

Carvalho, L. F., \& Primi, R. (2015). Development and internal structure investigation of the Dimensional Clinical Personality Inventory. Psicologia: Reflexão e Crítica, 28(2), 322330. https://doi.org/10.1590/1678-7153.201528212

Castro, M. M. L. D., Hökerberg, Y. H. M., \& Passos, S. R. L. (2013). Validade dimensional do instrumento de qualidade de vida WHOQOL-BREF aplicado a trabalhadores de saúde. Cadernos de Saúde Pública, 29(7), 1357-1369. https://dx.doi.org/10.1590/S0102-311X2013000700010

Chioqueta, A. P., \& Stiles, T. C. (2005). Personality traits and the development of depression, hopelessness, and suicide ideation. Personality and Individual Differences, 38(6), 1283-1291. https://doi.org/10.1016/j.paid.2004.08.010 


\section{H NTERAC̄öEM PSICOLOGIA}

Cramer, V., Torgersen, S., \& Kringlen, E. (2006). Personality disorders and quality of life. A population study. Comprehensive Psychiatry, 47(3), 178-184. https://doi.org/10.1016/ j.comppsych.2005.06.002

Crempien, C., Grez, M., Valdés, C., López, M. J., de la Parra, G., \& Krause, M. (2017). Role of personality functioning in the quality of life of patients with depression. The Journal of Nervous and Mental Disease, 205(9), 705-713. https:// doi.org/10.1097/NMD.0000000000000676

Fava, M., Alpert, J. E., Borus, J. S., Nierenberg, A. A., Pava, J. A., \& Rosenbaum, J. F. (1996). Patterns of personality disorder comorbidity in early-onset versus late- onset major depression. American Journal of Psychiatry, 153(10), 13081312. https://doi.org/10.1176/ajp.153.10.1308

Filipović, B. F., Randjelovic, T., Ille, T., Markovic, O., Milovanović, B., Kovacevic, N., \& Filipović, B. R. (2013). Anxiety, personality traits and quality of life in functional dyspepsia-suffering patients. European Journal of Internal Medicine, 24(1), 83-86. https://doi.org/10.1016/j.ejim.2012.06.017

Göktan, B., \& Akbağ, M. (2010). An investigation on Turkish military school students: Are there associations among big five personality factors, perceived family environment and hopelessness? Procedia - Social and Behavioral Sciences, 2(2), 5458-5462. https://doi.org/10.1016/j.sbspro. 2010.03.890

Hair Jr., J. F., Anderson, R. E., Tatham, R. L., \& Black, W. C. (2005). Análise multivariada de dados (5o ed). Bookman.

Herth, K. (1992). Abbreviated instrument to measure hope: Development and psychometric evaluation. Journal of Advanced Nursing, 17(10), 1251-1259. https://doi.org/ 10.1111/j.1365-2648.1992.tb01843.x

Hu, L., \& Bentler, P. M. (1999). Cutoff criteria for fit indexes in covariance structure analysis: Conventional criteria versus new alternatives. Structural Equation Modeling: A Multidisciplinary Journal, 6(1), 1-55. https://doi.org/ 10.1080/10705519909540118

Jonason, P. K., Foster, J. D., Csathó, Á., \& Gouveia, V. (2018). Expectancy biases underneath the Dark Triad traits: Associations with optimism, pessimism, and hopelessness. Personality and Individual Differences, 134, 190-194. https://doi.org/10.1016/j.paid.2018.06.020

Kotov, R., Gamez, W., Schmidt, F., \& Watson, D. (2010). Linking "big" personality traits to anxiety, depressive, and substance use disorders: A meta-analysis. Psychological Bulletin, 136(5), 768-821. https://doi.org/10.1037/a0020327

Marco, J. H., Guillén, V., \& Botella, C. (2017). The buffer role of meaning in life in hopelessness in women with borderline personality disorders. Psychiatry Research, 247, 120-124. https://doi.org/10.1016/j.psychres.2016.11.011
Millon, T. (2011). Disorders of Personality: Introducing a DSM/ ICD Spectrum from Normal to Abnormal ( $3^{a}$ ed.). John Wiley \& Sons, Inc. https://doi.org/ 10.1002/9781118099254.fmatter

Mutlu, T., Balbag, Z., \& Cemrek, F. (2010). The role of self-esteem, locus of control and big five personality traits in predicting hopelessness. Procedia - Social and Behavioral Sciences, 9, 1788-1792. https://doi.org/10.1016/j.sbspro. 2010.12.401

Penedo, F. J., Gonzalez, J. S., Dahn, J. R., Antoni, M., Malow, R., Costa, P., \& Schneiderman, N. (2003). Personality, quality of life and HAART adherence among men and women living with HIV/AIDS. Journal of Psychosomatic Research, 54(3), 271-278. https://doi.org/10.1016/s00223999(02)00482-8

Robison, E. J., Shankman, S. A., \& McFarland, B. R. (2009). Independent associations between personality traits and clinical characteristics of depression. The Journal of Nervous and Mental Disease, 197(7), 476-483. https:// doi.org/10.1097/NMD.0b013e3181aad5fc

Sartore, A. C., \& Grossi, S. A. A. (2008). Escala de Esperança de Herth: Instrumento adaptado e validado para a língua portuguesa. Revista da Escola de Enfermagem da USP, 42(2), 227-232. https://doi.org/10.1590/S008062342008000200003

Schrank, B., Amering, M., Hay, A. G., Weber, M., \& Sibitz, I. (2014). Insight, positive and negative symptoms, hope, depression and self-stigma: A comprehensive model of mutual influences in schizophrenia spectrum disorders. Epidemiology and Psychiatric Sciences, 23(3), 271-279. https://doi.org/10.1017/S2045796013000322

Sevilla-Llewellyn-Jones, J., Camino, G., Russo, D. A., Painter, M., Montejo, A. L., Ochoa, S., Jones, P. B., \& Perez, J. (2018). Clinically significant personality traits in individuals at high risk of developing psychosis. Psychiatry Research, 261, 498-503. https://doi.org/10.1016/j.psychres. 2018.01.027

Skodol, A. E., Clark, L. A., Bender, D. S., Krueger, R. F., Morey, L. C., Verheul, R., Alarcon, R. D., Bell, C. C., Siever, L. J., \& Oldham, J. M. (2011). Proposed changes in personality and personality disorder assessment and diagnosis for DSM-5 Part I: Description and rationale. Personality Disorders: Theory, Research, and Treatment, 2(1), 4-22. https:// doi.org/10.1037/a0021891

Tsanas, A., Saunders, K. E. A., Bilderbeck, A. C., Palmius, N., Osipov, M., Clifford, G. D., Goodwin, G. M. \& De Vos, M. (2016). Daily longitudinal self-monitoring of mood variability in bipolar disorder and borderline personality disorder. Journal of Affective Disorders, 205, 225-233. https:// doi.org/10.1016/j.jad.2016.06.065 
van der Wal, S. J., Bienvenu, O. J., Romanoski, A. J., Eaton, W. W., Nestadt, G., \& Samuels, J. (2018). Longitudinal relationships between personality disorder dimensions and depression in a community sample. Neurology, Psychiatry and Brain Research, 30, 56-61. https://doi.org/10.1016/ j.npbr.2018.05.005

Vrbova, K., Prasko, J., Latalova, K., Kamaradova, D., Ociskova, M., Marackova, M., \& Slepecky, M. (2017). Hope, self-stigma, personality traits and quality of life in patients with psychotic disorders. European Psychiatry, 41(Suppl), S389S390. https://doi.org/10.1016/j.eurpsy.2017.02.436
WHOQOL GROUP. (1998). Development of the World Health Organization WHOQOL-BREF Quality of Life Assessment. Psychological Medicine, 28(3), 551-558. https://doi.org/ 10.1017/S0033291798006667

Widiger, T. A., \& Costa, P. T. (Orgs.). (2013). Personality disorders and the five-factor model of personality (3rd ed.). American Psychological Association.

Data de submissão: 12/11/2018 Primeira decisão editorial: 08/04/2019 Aceite em 27/05/2019 